\title{
DISERTACIONES
}

ESTUDIOS

Para citar este artículo: Igartua, JJ., Guerrero-Martín, I., Cachón-Ramón, D., \& Rodríguez-de-Dios, I. (2018). Efecto de la similitud con el protagonista de narraciones contra el racismo en las actitudes hacia la inmigración. El rol mediador de la identificación con el protagonista. Anuario Electrónico de Estudios en Cominicación Social “Disertaciones”, 11(1), 56-75. Doi: http://dx.doi.org/10.12804/revistas.urosario.edu.co/ disertaciones/a.5272

\section{EFECTO DE LA SIMILITUD CON EL PROTAGONISTA DE NARRACIONES CONTRA EL RACISMO EN LAS ACTITUDES HACIA LA INMIGRACIÓN. EL ROL MEDIADOR DE LA IDENTIFICACIÓN CON EL PROTAGONISTA*}

\section{Effect of Similarity with the Protagonist of Narratives Against Racism in Attitudes Toward Immigration. The Mediating Role of Identification with the Protagonist \\ Efeito da similitude com o protagonista de narrações contra o racismo nas atitudes para a imigração. 0 rol mediador da identificação com o protagonista}

\author{
**Juan-José Igartua, Universidad de Salamanca (España) \\ jigartua@usal.es \\ Iñigo Guerrero-Martín, Universidad de Salamanca (España) \\ i.guerrero@usal.es \\ Diego Cachón-Ramón, Universidad de Salamanca (España) \\ diego.cachon@usal.es
}

* La realización de la investigación que se presenta se ha desarrollado en el marco del proyecto de investigación: "Herramientas narrativas para reducir el prejuicio. Efectos de la similitud, el contacto imaginado, la empatía y la voz narrativa" (referencia CSO2015-67611-P), dirigido por el Dr. Juan-José Igartua y financiado por el Ministerio de Economía y Competitividad (Programa Estatal de Fomento de la Investigación Científica y Técnica de Excelencia, Subprograma Estatal de Generación de Conocimiento, Plan Estatal de Investigación Científica y Técnica y de Innovación 2013-2016).

** Envíese la correspondencia sobre el artículo a este autor. 


\section{DISERTACIONES}

ESTUDIOS

Isabel Rodríguez-de-Dios, Universidad de Salamanca (España)

isabelrd@usal.es

\section{RESUMEN}

El principal objetivo de la presente investigación fue generar nuevo conocimiento en torno a los procesos de persuasión narrativa y su aplicación para reducir el prejuicio hacia inmigrantes altamente estigmatizados. Se analizó el efecto de la similitud entre el protagonista de una narración diseñada para reducir el prejuicio y la audiencia del mensaje en la identificación con el protagonista y en las actitudes hacia la inmigración. Para ello se llevó a cabo una investigación experimental en la que participaron 90 estudiantes de universidad. Como estímulos experimentales se utilizaron narraciones cortas escritas en primera persona que relataban la experiencia migratoria de un inmigrante estigmatizado. Los participantes fueron asignados aleatoriamente a dos condiciones experimentales diseñadas en función de cómo aparecía caracterizado el protagonista de la narración. Los resultados mostraron que la similitud influía positivamente en la identificación con el protagonista lo que se asociaba, a su vez, a actitudes más favorables hacia la inmigración. La investigación realizada abre una línea de estudio sobre los factores vinculados con la construcción de los personajes y de los mensajes narrativos, y su incidencia en procesos de recepción (identificación, enganche narrativo), e indirectamente, en variables vinculadas con el cambio social y la promoción de la tolerancia.

Palabras clave: persuasión narrativa, identificación con los personajes, prejuicio, inmigración, análisis mediacional.

\section{ABSTRACT}

The main goal of this research was to generate new knowledge about the processes of narrative persuasion and its application to reduce the prejudice towards highly stigmatized immigrants. We analyzed the effect of similarity between the protagonist of a narrative designed to reduce the prejudice and the audience of the message, in the identification with the protagonist and in the attitudes towards the immigration. For this, an experimental research was conducted in which 90 university students participated. As experimental stimuli, short stories written in first person that recounted the migratory experience of a stigmatized immigrant were used. Participants were randomly assigned to two experimental conditions, designed according to how the protagonist of the narration appeared. The results showed that the similarity positively influenced the identification with the protagonist which, in turn, was associated with more favorable attitudes toward immigration. This research opens a line of study on the factors related to the construction of characters and narrative messages, and their incidence in reception processes (identification, narrative engagement), and indirectly, in variables related to social change and the promotion of tolerance. 


\section{DISERTACIONES}

ESTUDIOS

Estudios de audiencias y recepción: audiencias minoritarias y nuevas mediaciones

ISSN: 1856-9536

Doi: http://dx.doi.org/10.12804/revistas.urosario.edu.co/disertaciones/v11i1

Volumen 11, Número 1/ Enero-junio 2018

Versión PDF para imprimir desde

http://revistas.urosario.edu.co/index.php/disertaciones

Keywords: Narrative persuasion, identification with the characters, prejudice, immigration, mediational analysis.

\section{RESUMO}

O principal objetivo da presente pesquisa foi gerar novo conhecimento em torno aos processos de persuasão narrativa e a sua aplicação para reduzir o prejuízo para imigrantes altamente estigmatizados. Analisou-se o efeito da similitude entre o protagonista de uma narração desenhada para reduzir o prejuízo e a audiência da mensagem, $n$ identificação com o protagonista e nas atitudes para a imigração. Para isto, levou-se a cabo uma pesquisa experimental na que participaram 90 estudantes de universidade. Como estímulos experimentais se utilizaram narrações cortas escritas em primeira pessoa que relatavam a experiência migratória de um imigrante estigmatizado. Os participantes foram assignados aleatoriamente a duas condições experimentais, desenhadas em função de como aparecia caracterizado o protagonista da narração. Os resultados mostraram que a similitude influía positivamente na identificação com o protagonista o que se associava, à sua vez, a atitudes mais favoráveis para a imigração. A pesquisa realizada abre uma linha de estudo sobre os fatores vinculados com a construção das personagens e das mensagens narrativas, e a sua incidência em processos de recepção (identificação, conquista narrativa), e indiretamente, em variáveis vinculadas com a mudança social e a promoção da tolerância.

Palavras-chave: persuasão narrativa, identificação com as personagens, prejuízo, imigração, análise mediacional.

\section{Introducción}

El uso de narraciones se ha convertido en los últimos años en un potente recurso para persuadir a las personas (Frank, Murphy, Chatterjee, Moran \& Baezconde-Garbanati, 2015). Una narración es una secuencia de acontecimientos conectados causalmente, en los que intervienen una serie de personajes de cuya experiencia las personas pueden obtener una enseñanza (Hoeken, Kolthoff \& Sanders, 2016). Por tanto, las narraciones siempre entregan información a partir de la cual las personas pueden modificar o adaptar sus creencias, actitudes o comportamientos. La investigación sobre persuasión narrativa se relaciona con el estudio de los procesos o mecanismos que explican cómo las narraciones pueden cambiar actitudes, creencias o conductas (de Graaf, Hoeken, Sanders \& Beentjes, 2012; Green \& Brock, 2000; Hoeken \& Fikkers, 2014; Igartua, 2010; Igartua \& Barrios, 2012). En este campo se han realizado aportaciones relevantes tanto para identificar los mecanismos explicativos de la persuasión narrativa, como para indagar sobre los aspectos de los mensajes narrativos que pueden incrementar su eficacia persuasiva. Este conocimiento está siendo utilizado para el desarrollo de intervenciones en comunicación en el campo de la salud y en la resolución de problemas sociales (Cohen, Tal-Or \& Mazor-Tregerman, 2015; Igartua \& Vega, 2016; Murphy, Frank, Chatterjee \& Baezconde-Garbanati, 2013; Tal-Or \& Tsfati, 2016; Wojcieszak \& Kim, 2016).

El objetivo principal del presente trabajo es generar nuevo conocimiento en torno a los procesos de persuasión narrativa, entendiendo que los mensajes narrativos pueden convertirse en herramientas para mejorar las 


\section{DISERTACIONES}

ESTUDIOS

Estudios de audiencias y recepción: audiencias minoritarias y nuevas mediaciones

ISSN: 1856-9536

Doi: http://dx.doi.org/10.12804/revistas.urosario.edu.co/disertaciones/v11i1

Volumen 11, Número 1 / Enero-junio 2018

Versión PDF para imprimir desde

http://revistas.urosario.edu.co/index.php/disertaciones

actitudes hacia colectivos estigmatizados como los inmigrantes en el contexto de la Unión Europea y en España, en particular. Para ello, en la investigación experimental que se presenta, se analizó el efecto de la similitud en términos de identidad social en la identificación con el protagonista de una narración, un inmigrante perteneciente a un colectivo estigmatizado que relata en primera persona su experiencia vital como inmigrante y muestra su preocupación por las actitudes xenófobas. Igualmente, se analizó el papel mediador de la identificación para explicar el efecto de la similitud en la mejora de las actitudes hacia los inmigrantes estigmatizados. Nuestro trabajo tiene una dimensión teórica (avanzar el conocimiento sobre los procesos de persuasión narrativa) y también aplicada (aportar evidencias sobre los factores que pueden incrementar la eficacia de las campañas contra el racismo).

\section{Contacto mediático intergrupal y reducción del prejuicio}

La mejora de las actitudes intergrupales hacia colectivos estigmatizados puede favorecerse por medio de actividades de contacto interpersonal, tal como se desprende de la hipótesis de contacto (Pettigrew \& Tropp, 2006). Sin embargo, también se ha observado que los efectos positivos del contacto pueden darse aunque este sea indirecto, vicario o meramente simbólico (Park, 2012). Por ejemplo, los formatos narrativos (un relato corto, una serie de televisión o un largometraje) ofrecen un contexto no amenazante en el cual los individuos pueden experimentar contacto parasocial vicario con personajes pertenecientes a exogrupos discriminados, que en situaciones de contacto social directo o interpersonal podrían causar incomodidad o inquietud (Chung \& Slater, 2013).

Park (2012) define el contacto intergrupal mediático, como aquella interacción parasocial que se produce entre un espectador que pertenece al endogrupo (por ejemplo, autóctono o nativo) y un personaje de una narración que forma parte de un exogrupo (un inmigrante o extranjero). La investigación en este campo también se ha referido a este proceso como contacto parasocial: las personas desarrollan relaciones parasociales con miembros de exogrupos que encuentran en los medios, y estas relaciones funcionan de manera similar a las relaciones reales (Schiappa, Gregg \& Hewes, 2005).

En el contexto de la investigación sobre reducción del prejuicio a través de contenidos mediáticos, Park (2012) plantea que la identificación con los personajes del exogrupo constituye un proceso relevante que explica el efecto prosocial del contacto mediático intergrupal. Así, se ha observado que el visionado de series de televisión con una imagen positiva de las minorías y la identificación con los personajes minoritarios se asociaba a actitudes más positivas hacia las minorías (Ortiz \& Harwood, 2007). En segundo lugar, en un estudio experimental realizado en Holanda sobre el impacto de una serie televisiva con un mensaje favorable al contacto multicultural, se observó que la exposición a la serie dramática multicultural (en comparación con el visionado de una serie que actuó como control) reducía la percepción de amenaza intercultural y la identificación con los personajes del exogrupo explicaba dicho efecto (Müller, 2009).

\section{Persuasión narrativa e identificación con los personajes}

La investigación sobre persuasión narrativa también es relevante en la investigación que se presenta, ya que se emplearon como estímulos experimentales narraciones cortas escritas en primera persona que relataban la experiencia migratoria de un inmigrante estigmatizado, desde su llegada a España hasta la actualidad. La investigación 


\section{DISERTACIONES}

ESTUDIOS

Estudios de audiencias y recepción: audiencias minoritarias y nuevas mediaciones

ISSN: 1856-9536

Doi: http://dx.doi.org/10.12804/revistas.urosario.edu.co/disertaciones/v11i1

Volumen 11, Número 1 / Enero-junio 2018

Versión PDF para imprimir desde

http://revistas.urosario.edu.co/index.php/disertaciones

sobre persuasión narrativa estudia los procesos o mecanismos que explican cómo las narraciones pueden cambiar actitudes, creencias o comportamientos (de Graaf et al., 2012; Green \& Brock, 2000; Moyer-Gusé, 2008; Slater \& Rouner, 2002). Una revisión del meta-análisis ha demostrado que las narraciones provocan efectos significativos en las actitudes, creencias, intención de conducta y comportamiento (Braddock \& Dillard, 2016).

Los personajes constituyen un ingrediente básico de cualquier narración y por ello su diseño o sus características pueden condicionar los procesos de recepción e impacto persuasivo. En el campo de la persuasión narrativa se ha puesto de manifiesto que la identificación con los personajes juega un importante rol mediador: para que una narración provoque un impacto persuasivo es necesario que se produzca una alta identificación con los personajes que la protagonizan (Cohen et al., 2015; de Graaf et al., 2012; Hoeken \& Fikkers, 2014; Igartua, 2010; Igartua \& Barrios, 2012; Igartua \& Vega, 2016; Tal-Or \& Tsfati, 2016). En este contexto, la identificación con los personajes constituye un proceso cognitivo-afectivo que tiene lugar durante la recepción (lectura, visionado) del mensaje narrativo y que se vincula con la toma de perspectiva o empatía cognitiva - ponerse en el lugar del personaje-, la empatía emocional -sentir las mismas emociones que el personaje-y la pérdida temporal de la autoconciencia -el receptor de la narración imagina que es el personaje, asume su identidad lo que produce un proceso de merging o fusión - (Cohen, 2001; Igartua \& Barrios, 2012; Moyer-Gusé, 2008).

La identificación proporciona una oportunidad para probar otras identidades y adoptar los sentimientos y pensamientos de otro. Considerada de este modo, la identificación permite al individuo superar la tendencia natural a limitar la visión de las cosas a una sola perspectiva asumiendo el punto de vista del personaje. Por otro lado, los modelos más relevantes para el estudio de los procesos de la persuasión narrativa (Extended Elaboration Likelihood Model, E-ELm, Slater \& Rouner, 2002; Entertainment Overcoming Resistance Model, EORM, Moyer-Gusé, 2008) plantean que las narraciones son instrumentos eficaces para debilitar actitudes fuertemente consolidadas, incluso cuando se abordan temas controvertidos (Cohen et al., 2015; Igartua \& Barrios, 2012), porque se asume que la identificación disminuye la contra-argumentación. Ello quiere decir que cuando las personas se identifican con los personajes, su capacidad de generar críticas a los mensajes actitudinales de la narración se reduce y, por lo tanto, su resistencia a la persuasión se debilita. Además, la identificación permite a las personas asumir el punto de vista y la perspectiva del personaje, lo que aumenta la probabilidad de que las personas acepten las creencias y actitudes implícitas en dichas narraciones o vehiculadas por las afirmaciones realizadas por los personajes. En este contexto, la investigación empírica ha encontrado que cuando una persona se identifica con el protagonista de una narración, esta asume la perspectiva de dicho personaje desde un punto de vista cognitivo, lo que lleva a cambios en las creencias u opiniones (de Graaf et al., 2012; Igartua \& Barrios, 2012).

La aplicación de estos hallazgos al diseño de mensajes narrativos para ser utilizados en campañas contra el racismo, cuyos protagonistas son miembros de exogrupos estigmatizados, plantea dos desafíos. Por un lado, los personajes estigmatizados provocan menos identificación (Chung \& Slater, 2013). En segundo lugar, los individuos con un alto grado de prejuicio, es decir, aquellos que pueden beneficiarse especialmente de la exposición a mensajes positivos hacia los exogrupos estigmatizados, son menos propensos a identificarse con los personajes que representan a miembros de dichos grupos (Igartua \& Frutos, 2017). 


\section{DISERTACIONES}

ESTUDIOS

Estudios de audiencias y recepción: audiencias minoritarias y nuevas mediaciones

ISSN: 1856-9536

Doi: http://dx.doi.org/10.12804/revistas.urosario.edu.co/disertaciones/v11i1

Volumen 11, Número 1 / Enero-junio 2018

Versión PDF para imprimir desde

http://revistas.urosario.edu.co/index.php/disertaciones

\section{Similitud, identificación con los personajes e impacto persuasivo}

En la definición aportada por Cohen (2001) se enfatiza que la identificación es un proceso imaginativo, es decir, que para que se produzca identificación con un personaje la persona expuesta a una narración ha de poder imaginar que en ese momento se siente como si fuera dicho personaje. Ahora bien, existe escasa investigación sobre los factores de construcción de los personajes que incrementan la identificación e, indirectamente, el impacto persuasivo. Una línea de investigación en este campo está relacionada con el efecto de la similitud entre el protagonista de la narración y la audiencia en la identificación y en la efectividad persuasiva del relato (de Graaf, 2014; Hoeken et al., 2016).

La similitud describe un proceso a través del cual el receptor de la narración evalúa hasta qué punto comparte un determinado rasgo con el protagonista del relato. La similitud se puede establecer en rasgos objetivos (como los aspectos demográficos, el sexo o la nacionalidad) pero también en características psicológicas o subjetivas (como la personalidad, las creencias u opiniones, los valores o la identidad social). Se asume que tanto la similitud percibida como la similitud en atributos objetivos incrementan la identificación e, indirectamente, influyen en las actitudes. En este sentido, la similitud es un prerrequisito para que se produzca la identificación con un personaje, puesto que la identificación implica que se produzca una fusión de identidades (Cohen, 2001).

La investigación previa sobre el efecto de la similitud en el impacto persuasivo se ha realizado con historias o relatos cortos escritos. La similitud se ha manipulado experimentalmente variando las características del protagonista del relato y teniendo en cuenta al mismo tiempo la situación o características de los participantes (Chen, Bell \& Taylor, 2016; de Graaf, 2014; Hoeken et al., 2016). En el trabajo de de Graaf (2014) se manipuló la información sobre dónde vivía la protagonista de la narración (con sus padres o en una residencia de estudiantes); al mismo tiempo, se preguntaba a los participantes con quién vivían. Se consideraba que existía una alta similitud si había coincidencia entre el lugar de residencia del personaje y el lugar de residencia de la persona que leía la historia. En este caso, se manipuló la similitud en términos demográficos (lugar de residencia). La narración se centraba en temas de salud (la protagonista tenía cáncer y se describía cómo hacía frente a la enfermedad) y el objetivo persuasivo era incrementar la percepción de riesgo. Los resultados mostraron que la similitud con el personaje influía en la similitud percibida y, además, incrementaba la percepción de riesgo. Por tanto, la similitud ejercía un efecto actitudinal congruente con el contenido de la narración. Ahora bien, de Graaf et al. (2016) observaron que solo en dos de un total de once estudios revisados, se comprobó que la similitud incrementaba el impacto actitudinal.

Una limitación de la investigación previa sobre los efectos de la similitud es que se ha centrado exclusivamente en la similitud demográfica (por ejemplo, si el personaje y la audiencia comparten características como el género, la edad o el grupo étnico; véase de Graaf et al., 2016). Por otro lado, la cuestión crucial a la que nosotros hacemos frente es cómo fomentar la identificación con un personaje inmigrante estigmatizado, protagonista de una narración destinada a mejorar las actitudes intergrupales ya que la investigación previa ha observado que es difícil inducir identificación con personajes estigmatizados (Chung \& Slater, 2013; Igartua \& Frutos, 2017). Sostenemos que es necesario trabajar sobre otras dimensiones de la similitud y, en concreto, asumimos que estimular la similitud en términos de identidad social es una manera adecuada de promover la identificación con un personaje inmigrante estigmatizado e, indirectamente, mejorar las actitudes hacia el exogrupo. En otras palabras, si 


\section{DISERTACIONES}

ESTUDIOS

Estudios de audiencias y recepción: audiencias minoritarias y nuevas mediaciones

ISSN: 1856-9536

Doi: http://dx.doi.org/10.12804/revistas.urosario.edu.co/disertaciones/v11i1

Volumen 11, Número 1 / Enero-junio 2018

Versión PDF para imprimir desde

http://revistas.urosario.edu.co/index.php/disertaciones

el protagonista de dicha narración indica que comparte intereses, pasatiempos y sentimientos nacionales con la audiencia autóctona, es decir, si el inmigrante se presenta con un perfil de alta similitud en términos de identidad social, los individuos del grupo dominante (autóctonos) tendrán más probabilidades de identificarse con dicho personaje y, por ello, será más probable que se induzca un impacto en actitudes e intención de conducta.

\section{Contexto de la investigación e hipótesis}

El asentamiento de población inmigrante ha modificado considerablemente el mapa demográfico de la sociedad española, que se dirige hacia un modelo cada vez más heterogéneo y multicultural. Dicho proceso ha contribuido al desarrollo económico, social y cultural del país. Sin embargo, el actual contexto de crisis económica es caldo de cultivo para que tomen fuerza posturas extremistas y de rechazo hacia la población extranjera. En este sentido, según la última encuesta publicada hasta la fecha sobre actitudes hacia la inmigración, desarrollada por el Centro de Investigaciones Sociológicas (cIs), el 70,5\% de los ciudadanos consultados considera que el número de inmigrantes en España es "excesivo" o "elevado" y únicamente el 20,9\% que es "aceptable" y ello, a pesar de que apenas supone el $10 \%$ de la población española. Además, el 47,2\% de las personas entrevistadas considera que los españoles tratan a los inmigrantes con desconfianza, indiferencia, desprecio o agresividad (cIs, 2014). En este contexto, es prioritario desarrollar estrategias que reduzcan el prejuicio por parte de la población autóctona hacia los inmigrantes con el objetivo de promover el desarrollo de sociedades más inclusivas.

En la presente investigación se analizó el efecto de la similitud en términos de identidad social en la identificación con el protagonista de una narración que relataba en primera persona su experiencia vital como inmigrante y mostraba su preocupación por las actitudes xenófobas. Igualmente, se analizó el papel mediador de la identificación para explicar el efecto de la similitud en las actitudes hacia inmigrantes estigmatizados. Para ello se realizó un estudio experimental en el que participaron estudiantes universitarios que fueron asignados aleatoriamente a dos condiciones experimentales diseñadas en función de cómo aparecía caracterizado el protagonista de una narración escrita en primera persona y, pensada para reducir el prejuicio hacia inmigrantes estigmatizados. La manipulación experimental de la narración estaba planteada para estimular una alta o baja similitud en términos de identidad social.

La primera hipótesis se relaciona con el impacto de la similitud en la identificación con el protagonista de la narración. Se esperaba que la lectura de una narración diseñada para reducir el prejuicio, protagonizada por un inmigrante estigmatizado (de origen marroquí) que presentaba alta similitud en términos de identidad social, induciría una mayor identificación con el personaje, en comparación con la lectura de una narración protagonizada un inmigrante que presentaba una baja similitud. De este modo, se formuló la primera hipótesis:

\section{H1: La similitud en términos de identidad social influirá positivamente en la identificación con el protagonista de la narración.}

La segunda hipótesis se refiere al efecto de la similitud en las actitudes e intención de conducta, es decir, en la mejora de las actitudes intergrupales. Se asumía que la lectura de una narración protagonizada por un inmigrante estigmatizado pero caracterizado para presentar una alta similitud en términos de identidad social, en compara- 


\section{DISERTACIONES}

ción con la lectura de una narración protagonizada un inmigrante que presentaba una baja similitud, provocaría un efecto positivo en las actitudes hacia la inmigración y en la intención de conducta de aproximación hacia inmigrantes marroquíes:

\section{H2: La similitud en términos de identidad social influirá positivamente en las actitudes hacia la inmigración ( $\mathrm{H} 2 \mathrm{a}$ ) y en la intención de conducta de aproximación hacia inmigrantes marroquíes (H2b).}

Finalmente, se esperaba contrastar que la identificación con el protagonista de la narración diseñada para mejorar las actitudes intergrupales actuara como variable mediadora, es decir, que se produjera un efecto indirecto de la similitud en las actitudes hacia la inmigración e intención de conducta de aproximación hacia inmigrantes marroquíes a través de la identificación con el protagonista.

H3: La similitud en términos de identidad social influirá positivamente en la identificación con el protagonista de la narración, lo que se asociará a su vez a una actitud positiva hacia la inmigración (H3a) y a una mayor intención de conducta de aproximación hacia inmigrantes marroquíes (H3b) (figura 1).

Figura 1. Modelo de mediación hipotetizado (H3)

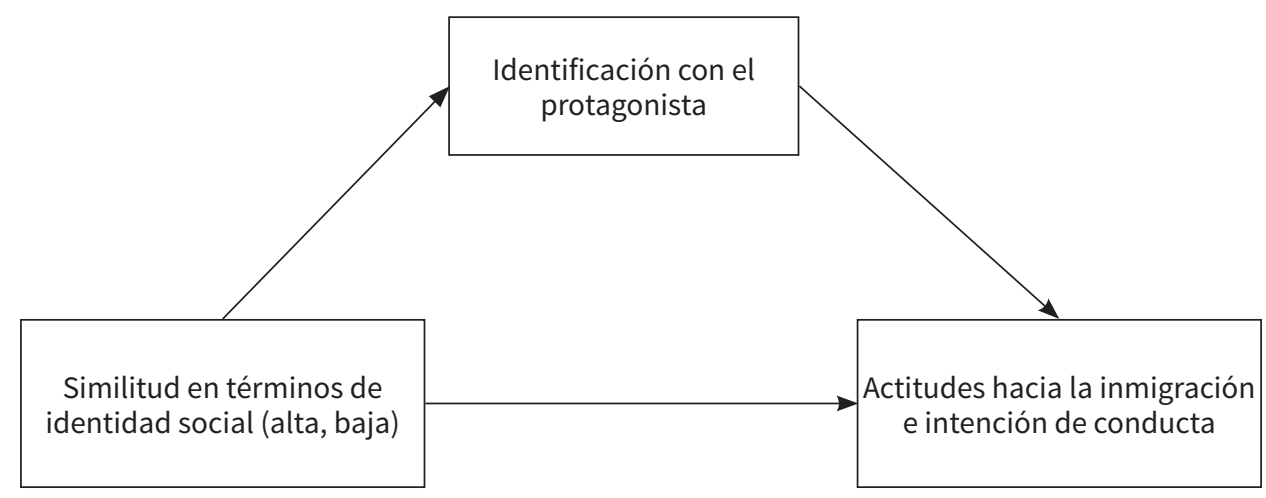

\section{Método}

\section{Participantes}

En la investigación participaron 90 estudiantes de la Universidad de Salamanca, de nacionalidad española, pertenecientes a diferentes titulaciones y que participaron de manera voluntaria sin recibir ningún incentivo por ello. El $57,8 \%$ de los participantes eran mujeres y el $42,2 \%$ hombres y la media de edad fue de 21,47 años ( $D T=2,91$, rango: 18 a 34 años). 


\section{DISERTACIONES}

ESTUDIOS

Estudios de audiencias y recepción: audiencias minoritarias y nuevas mediaciones

ISSN: 1856-9536

Doi: http://dx.doi.org/10.12804/revistas.urosario.edu.co/disertaciones/v11i1

Volumen 11, Número 1 / Enero-junio 2018

Versión PDF para imprimir desde

http://revistas.urosario.edu.co/index.php/disertaciones

\section{Diseño y procedimiento}

Se realizó una investigación experimental con un diseño aleatorio de dos grupos con solo medida postest, siendo la variable independiente la similitud con el protagonista de la narración (alta, baja) en términos de identidad social. Los participantes fueron asignados aleatoriamente a las dos condiciones experimentales, se les informaba que tenían que leer una narración y posteriormente contestar a una serie de preguntas mediante un cuestionario.

Tomando como referencia estudios previos y noticias aparecidas en los medios comunicación, se elaboró una narración escrita en la cual un inmigrante marroquí relataba su experiencia migratoria en España y cuyo objetivo era la reducción del prejuicio hacia los inmigrantes. La narración estaba escrita en primera persona, ya que la investigación previa sobre los efectos de la voz narrativa ha documentado que las narraciones en primera persona son más efectivas que las narraciones escritas en tercera persona tanto para incrementar la identificación con los personajes como para producir un efecto en las actitudes e intención de conducta (de Graaf et al., 2012; de Graaf, Sanders, \& Hoeken, 2016; Kaufman \& Libby, 2012).

Se optó por narrar la experiencia de un inmigrante de origen marroquí porque constituye en la actualidad uno de los colectivos inmigrantes más estigmatizados, que genera menos simpatía en España (junto con los rumanos) y, además, es el colectivo que se identifica de manera más inmediata como inmigrante en comparación con otras nacionalidades (cIs, 2014). En la narración, Saîd (nombre ficticio) aludía a diferentes hechos, circunstancias y sentimientos relacionados con la llegada al país, las primeras actividades laborales, la situación laboral actual, su situación familiar, sus sentimientos relacionados con el hecho de vivir en España, su vida social y la crianza de sus hijos, el manejo o dominio del idioma y su sentimiento de pertenencia. También mencionaba dos cuestiones relacionadas con el rechazo a los inmigrantes: la alusión a que mucha gente piensa que los inmigrantes quitan el trabajo a los españoles autóctonos y la alusión a que muchas personas consideran que existe una relación entre inmigración y criminalidad. En ambos casos, el protagonista de la narración reclamaba una mayor tolerancia hacia los inmigrantes planteando medidas como perseguir la explotación laboral y desarrollar campañas y acciones educativas contra el racismo y la xenofobia.

La manipulación experimental de la narración estaba diseñada para estimular una alta (506 palabras) o baja similitud (523 palabras) con los participantes del estudio en términos de identidad social. Para reforzar la similitud con el protagonista de la narración se tuvieron en cuenta diversos aspectos: el hecho de sentirse español (versus marroquí en la condición de baja similitud), mencionar que en su negocio trabajan españoles (versus inmigrantes marroquíes), informar que se tiene sobre todo amigos españoles (versus marroquíes), indicar que su plato favorito es de origen español (versus marroquí), comentar que con los hijos se habla habitualmente en español (versus árabe) y que se leen sobre todo periódicos españoles (versus marroquíes), indicar que se desea seguir viviendo en España (versus, regresar a Marruecos), y mencionar que se identifica con la cultura española (versus marroquí) y con su bandera. Para reforzar la manipulación experimental, la narración venía acompañada por una fotografía de Saîd en su habitación, donde en una de las paredes había una bandera española (versus marroquí).

Se realizaron dos estudios piloto, el primero de ellos para efectuar la selección de la fotografía que acompañaba la narración y el segundo para contrastar si la manipulación de la similitud era eficaz. Para realizar el primer estudio piloto se contó con una muestra de estudiantes ( $N=21$, de nacionalidad española) que valoraron tres fotografías de personas de origen marroquí (tomadas de fondos fotográficos sin derechos de autor) en términos 


\section{DISERTACIONES}

ESTUDIOS

Estudios de audiencias y recepción: audiencias minoritarias y nuevas mediaciones

ISSN: 1856-9536

Doi: http://dx.doi.org/10.12804/revistas.urosario.edu.co/disertaciones/v11i1

Volumen 11, Número 1 / Enero-junio 2018

Versión PDF para imprimir desde

http://revistas.urosario.edu.co/index.php/disertaciones

de atractivo, aspecto agradable, honestidad, carácter amenazante y carácter amistoso (con escalas de 0 a 10). Se seleccionó la fotografía que obtuvo mejores puntuaciones en dichos rasgos.

Por medio de una segunda muestra de estudiantes españoles $(N=54)$ se evaluó también si existían diferencias entre las dos versiones de la narración en las siguientes variables "el mensaje es claro y comprensible", "el mensaje es creíble" y "el mensaje es interesante" (desde 1 "muy en desacuerdo", hasta 7 "muy de acuerdo"). En las tres variables se obtuvieron resultados similares, no observándose diferencias significativas (con medias superiores a 5 en todos los casos). Finalmente, también se pidió a los participantes que valoraran: "en qué medida consideras que tú tienes algunas cosas en común con Saîd" (desde 0 "nada" a 10 "mucho"). Los participantes que habían leído la narración de alta similitud con el protagonista $(n=28)$ puntuaban significativamente más alto en dicho ítem $(M=4,96, D T=2.49)$ que los que habían leído la narración diseñada para estimular baja similitud $(n=26, M=$ $3,35, D T=2,99)(t(48,80)=-2,009, p<0,05)$.

\section{Instrumentos y variables}

Se utilizó un cuestionario autoadministrado con portada (donde se indicaban los objetivos del estudio), narración (diferente en cada condición experimental) y una serie de escalas para analizar los procesos de recepción (transporte narrativo, identificación con el protagonista, realismo percibido) e impacto en actitudes e intención de conducta. Además, se incluyeron dos preguntas para contrastar la eficacia de la manipulación experimental. Finalmente, también se tomaron medias sobre las variables sociodemográficas (sexo, edad), autoposicionamiento político y contacto directo con inmigrantes marroquíes, cuyo rol era comprobar la eficacia del proceso de aleatorización (la equivalencia de los grupos experimentales).

Percepción de similitud con el protagonista de la narración. Como variable de chequeo de la manipulación experimental se preguntó a los participantes inmediatamente después de leer la narración estas dos preguntas: “ien qué medida consideras que tú tienes algunas cosas en común con Saîd?" y “¿cuánto crees que te pareces a Saîd?” (desde 0 $=$ nada, hasta 10 = mucho). Como ambos ítems estaban fuertemente correlacionados $(r(88)=0,77, p<0,001)$ se combinaron para formar un índice de similitud percibida con el protagonista ( $\alpha=0,87 ; M=4,46, D T=2,54)$.

Identificación con el protagonista. Se evaluó con una escala, que ya había sido validada en estudios previos (Igartua \& Barrios, 2012), compuesta por 11 ítems (ejemplos de ítems, "me he sentido implicado afectivamente con los sentimientos de Saîd", "me he sentido como si yo fuera Saîd", "he intentado imaginar los sentimientos, pensamientos y reacciones de Saîd", "he tenido la impresión de vivir realmente yo mismo la historia de Saîd"; desde $1=$ nada, hasta $5=$ mucho; $\alpha=0,87, M=3,42, D T=0,64$ ).

Transporte narrativo. Se evaluó con una escala desarrollada a partir de estudios previos (Green \& Brock, 2000; Wojcieszak \& Kim, 2016), compuesta por 8 ítems que miden el grado de absorción o enganche con la narración (ejemplo de ítems: "me sentí muy involucrado o implicado con la narración", "después de terminar la lectura, sentía que quería saber más cosas acerca de la situación de personas como el protagonista de la narración”, desde $1=$ totalmente en desacuerdo, hasta $7=$ totalmente de acuerdo). Un análisis factorial de componentes principales extrajo dos factores que explicaban el 63,38\% de la varianza. El primer factor hacía referencia al enganche narrativo ("la narración me ha afectado emocionalmente"; $\alpha=0,84, M=4,59, D T=1,15$ ) y el segundo factor hacía referencia a la lectura distraída ("mientras leía el relato estaba pensando en otras cosas"; $\alpha=0,72, M=1,69, D T=0,82$ ). 


\section{DISERTACIONES}

ESTUDIOS

Estudios de audiencias y recepción: audiencias minoritarias y nuevas mediaciones

ISSN: 1856-9536

Doi: http://dx.doi.org/10.12804/revistas.urosario.edu.co/disertaciones/v11i1

Volumen 11, Número 1 / Enero-junio 2018

Versión PDF para imprimir desde

http://revistas.urosario.edu.co/index.php/disertaciones

Realismo percibido de la narración. Se utilizó una escala compuesta por 4 ítems (Cho, Shen, \& Wilson, 2014): "en la narración se describían situaciones que podrían suceder en la vida real", "lo que le sucede Saîd es lo que suele pasar a los inmigrantes en el mundo real en España", "el testimonio de Saîd era realista" y "la historia contada por Saîd tenía sentido" (desde 1 = totalmente en desacuerdo, hasta 7 = totalmente de acuerdo; $\alpha=0,85, M=$ $5,75, D T=1,13)$.

Actitudes hacia la inmigración. Teniendo en cuenta los argumentos expresados en la narración por el protagonistas de la misma, se creó una escala ad hoc compuesta por 3 ítems: "¿en qué medida estás a favor o en contra de la inmigración?", "¿en qué medida estás a favor o en contra de que el Gobierno español financie campañas de información sobre los inmigrantes (por ejemplo, contra el racismo)?" y “ien qué medida estás a favor o en contra de que el Gobierno fortalezca las leyes para impedir la explotación de los inmigrantes en los lugares de trabajo en España?" (desde desde 1 = estoy totalmente en contra, hasta 7 = estoy totalmente a favor). Un análisis factorial de componentes principales extrajo un único factor que explicaba el 62,14\% de la varianza, por lo que los tres ítems se combinaron para crear un índice de actitud hacia la inmigración ( $\alpha=0,69, M=5,99, D T=1,04)$.

Intención de conducta. Se evalúo el deseo de entablar en el futuro contacto con inmigrantes marroquíes mediante una escala formada por 3 ítems (Crisp \& Husnu, 2011): "pensando en la próxima vez que te encuentres en una situación en donde podrías interactuar con un inmigrante marroquí (por ejemplo, haciendo cola en la parada de autobús), ¿en qué medida estarías interesado en entablar una conversación con dicha persona? (desde $1=$ nada interesado, hasta $7=$ muy interesado), ¿en qué medida consideras que es importante para ti aprender más acerca de los inmigrantes marroquíes y sobre los problemas a los que se enfrentan? (desde 1 = nada importante, hasta 7 = muy importante) y ¿en qué medida estarías interesado en participar en un grupo de discusión que incluyera inmigrantes marroquíes y donde la conversación se centrara en los temas del prejuicio y la discriminación? (desde 1 = nada interesado, hasta 7 = muy interesado). Un análisis factorial de componentes principales extrajo un único factor que explicaba el $61,71 \%$ de la varianza, por lo que los tres ítems se combinaron para crear un índice de intención de conducta de aproximación hacia los inmigrantes marroquíes ( $\alpha=0,68, M=4,96, D T=1,21)$.

Contacto directo con inmigrantes marroquíes. Se preguntó a los participantes "¿cuánto contacto tienes en tu vida cotidiana con inmigrantes procedentes de Marruecos?" (desde 1 = nada, hasta $5=$ mucho; $M=2,40, D T=1,18$ ).

Autoposicionamiento político. Los participantes indicaron su ideología política con una escala de 11 puntos, desde 0 (izquierda) hasta 10 (derecha) $(M=3,83, D T=2,67)$.

\section{Resultados}

\section{Análisis preliminares}

En primer lugar se comprobó si existían diferencias estadísticamente significativas entre los dos grupos experimentales en las variables sociodemográficas (sexo, edad), autoposicionamiento político y contacto directo con inmigrantes marroquíes con el fin de comprobar si la aleatorización había sido realizada de manera eficaz. Se observó que no existían diferencias estadísticamente significativas entre los dos grupos experimentales en las variables sexo $\left(\chi^{2}(1, N=90)=0,18, p=0,670\right)$, edad $(t(88)=-0,86, p=0,388)$ y contacto directo con inmigrantes marroquíes ( $t(88)=0,53, p=0,597)$. Sin embargo, sí existían diferencias significativas en el autoposicionamiento 


\section{DISERTACIONES}

ESTUDIOS

Estudios de audiencias y recepción: audiencias minoritarias y nuevas mediaciones

ISSN: 1856-9536

Doi: http://dx.doi.org/10.12804/revistas.urosario.edu.co/disertaciones/v11i1

Volumen 11, Número 1 / Enero-junio 2018

Versión PDF para imprimir desde

http://revistas.urosario.edu.co/index.php/disertaciones

político ( $t(88)=-2,47, p<0,015)$. Puede decirse que la aleatorización fue exitosa, en términos generales. No obstante, dado que existían diferencias significativas en el autoposicionamiento político, en los siguientes análisis para el contraste de hipótesis dicha variable se incluyó como variable de control.

También se contrastó si la manipulación experimental de la similitud con el protagonista de la narración había sido efectiva. Mediante una prueba t de Student de medidas independientes se contrastó que efectivamente existían diferencias estadísticamente significativas en el índice de percepción de similitud $(t(88)=-3,29, p<0,001)$. Los participantes que habían leído la narración que presentaba a su protagonista como similar en términos de identidad social consideraban en mayor medida que Saîd era similar a ellos y que tenían cosas en común ( $M=5,30$, $D T=2,45)$, en comparación con los participantes que leyeron una narración donde el protagonista mostraba una baja similitud $(M=3,62, D T=2,37)$. Ahora bien, la manipulación de la similitud con el protagonista de la narración no influyó en la percepción de realismo de la narración $(t(88)=0,30, p=0,765)$ ni en el enganche narrativo $(t(88)=$ $-1,31, p=0,192)$ ni en el grado de distracción durante la lectura de la narración ( $t(87)=0,72, p=0,473)$.

Finalmente, se analizaron las correlaciones entre los procesos de recepción, observándose que la identificación se asociaba positivamente con el enganche narrativo y el realismo percibido, y negativamente con la distracción durante la lectura de la narración. El enganche narrativo se asociaba negativamente con la distracción y positivamente con el realismo percibido el cual, a su vez, se asociaba negativamente con la distracción (tabla 1).

Tabla 1. Correlaciones de Pearson entre los procesos de recepción

\begin{tabular}{|l|c|c|c|}
\hline & Distracción & Realismo percibido & Identificación \\
\hline Enganche narrativo &,$- 27^{\star \star}$ &, $54^{\star \star \star}$ &, $79^{\star \star \star}$ \\
\hline Distracción & &,$- 23^{\star}$ &,$- 33^{\star \star \star}$ \\
\hline Realismo percibido & & &, $57^{\star \star \star}$ \\
\hline
\end{tabular}

${ }^{\star} \mathrm{p}<, 05,{ }^{* *} \mathrm{p}<, 01,{ }^{* *} \mathrm{p}<, 001$

\section{Hipótesis 1: efecto de la similitud en la identificación con el protagonista}

La H1 planteaba que la similitud en términos de identidad social induciría una mayor identificación con el protagonista de la narración. Para contrastar dicha hipótesis se llevó a cabo un análisis de covarianza (Ancova) en el que se introdujo como covariable el autoposicionamiento político (figura 2).

Los resultados mostraron que, controlando estadísticamente el efecto del autoposicionamiento político, la narración protagonizada por un inmigrante marroquí que se presentaba como similar a la audiencia del mensaje en términos de identidad social provocó mayor identificaron $(M=3,58, E T=0,09)$ que la narración protagonizada por un inmigrante marroquí que presentaba baja similitud $(M=3,28, E T=0,09)\left(F(1,83)=5,06, p<0,027, \eta_{p}^{2}\right.$ $=0,058)$. También se observó que el efecto del autoposicionamiento político influía de manera significativa en la identificación con el protagonista de la narración $\left(F(1,83)=11,20, p<0,001, \eta_{p}{ }^{2}=0,119\right)$, mostrando ambas variables una correlación negativa $(r(84)=-0,29, p<0,003)$, lo que significa que las personas más conservadoras se identificaron menos con el protagonista de la narración. Estos resultados, por tanto, corroboran la hipótesis 1. 
Figura 2. Efecto de la similitud en términos de identidad social en la identificación con el protagonista

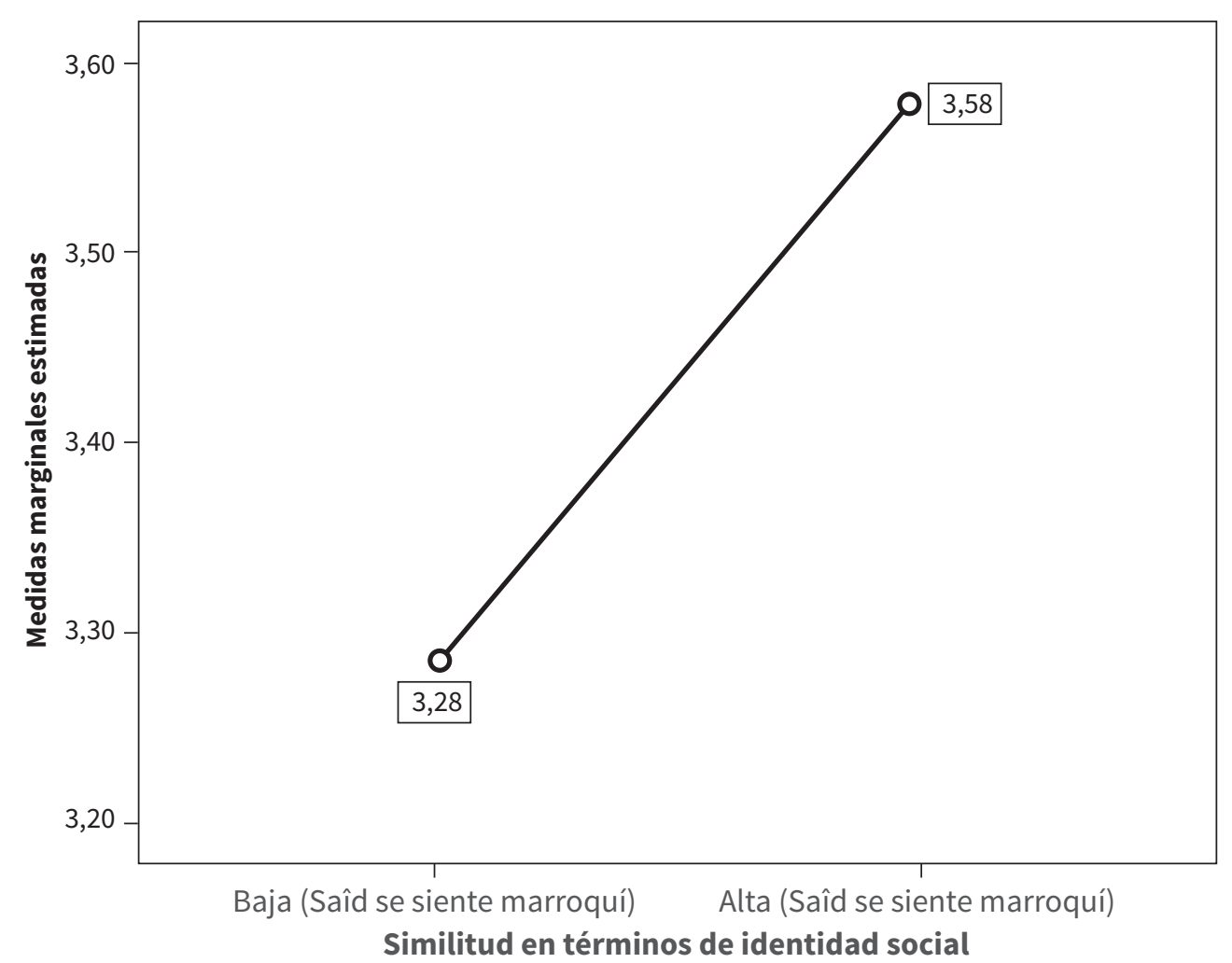

\section{Hipótesis 2: efecto de la similitud en las actitudes hacia la inmigración e intención de conducta}

La H2 planteaba que la similitud en términos de identidad social influiría positivamente en las actitudes hacia la inmigración y en la intención de conducta de aproximación hacia inmigrantes marroquíes. Para contrastar dicha hipótesis se ejecutaron dos análisis de covarianza (Ancova) incluyendo como covariable el autoposicionamiento político. Los resultados no confirmaron la hipótesis 2, ya que la similitud no influía de manera significativa en las actitudes favorables hacia la inmigración $(F(1,87)=1,14, p=0,288$, potencia observada $=0,18)$ ni en la intención de conducta $(F(1,87)=0,00, p=0,985$, potencia observada $=0,05)$. Nuevamente, se observó que el autoposicionamiento político ejercía un efecto significativo en las actitudes hacia la inmigración $\left(F(1,87)=13,34, p<0,001, \eta_{p}{ }^{2}=\right.$ $0,133 ; r(88)=-0,34, p<0,001)$ y en la intención de conducta de aproximación hacia los inmigrantes marroquíes $(F$ $\left.(1,87)=9,32, p<0,001, \eta_{p}^{2}=0,097 ; r(88)=-0,32, p<0,001\right)$.

\section{Hipótesis 3: análisis mediacional (efecto indirecto)}

El análisis de mediación es un método de análisis estadístico que permite responder a la pregunta sobre cuánto y cómo una variable independiente (en nuestro caso, la similitud) transmite su efecto sobre una variable dependiente (en el presente estudio, las actitudes hacia la inmigración y la intención de conducta). Para llevar a cambo 


\section{DISERTACIONES}

ESTUDIOS

dicho análisis mediacional y contrastar la H3, se utilizó la macro PROCESS para SPSS creada por Hayes (2013). Este procedimiento permite calcular los efectos directos, indirectos y totales. En estos análisis, el efecto directo ( $c^{\prime}$ ) cuantifica el efecto de la similitud sobre las actitudes hacia la inmigración y la intención de conducta cuando se controla estadísticamente la variable mediadora considerada (en este caso, la identificación con el protagonista de la narración). El efecto indirecto ( $a b)$ cuantifica el efecto de la similitud (variable independiente) sobre las actitudes hacia la inmigración y la intención de conducta (variables dependientes) a través de la identificación con el protagonista de la narración (variable mediadora). Finalmente, el efecto total (c) es la suma del efecto directo y el efecto indirecto $\left(c=c^{\prime}+a b\right)$.

Es preciso aclarar que a pesar de que se observó que la similitud no influía de manera significativa en las actitudes hacia la inmigración ni en la intención de conducta (efecto total no significativo), es posible que el efecto provocado por la similitud en dichas variables se produzca de manera indirecta a través de la mediación de la identificación con el protagonista de la narración, que es precisamente lo que se planteaba en la H3. De hecho, la estrategia recomendada en la actualidad para el análisis mediacional sugiere que dado que el efecto total es la suma del efecto directo e indirecto, el efecto indirecto puede ser estadísticamente significativo a pesar de que no se haya obtenido un efecto total significativo (Hayes, 2013).

El uso de la macro PROCESS se ha popularizado en la actualidad para la realización de análisis mediacionales -es decir, por qué o cómo una variable independiente ejerce un efecto en una o más variables dependientesporque permite la estimación de los efectos indirectos, los errores estándar y los intervalos de confianza con base en la distribución obtenida con la técnica de bootstrapping. Este método utiliza remuestreo con reemplazamiento, donde a partir de la muestra original se generan series de muestras simuladas para calcular el error típico (ET) asociado al coeficiente de regresión que cuantifica el efecto indirecto. En la presente investigación los efectos indirectos se calcularon para el modelo 4, utilizando 10000 muestras de bootstrapping, generando intervalos de confianza del tipo bias-corrected boostrap. En este contexto, se considera que un efecto indirecto es estadísticamente significativo si el intervalo de confianza establecido (IC al 95\%) no incluye el valor 0 . Si el valor 0 está incluido en dicho intervalo de confianza no se puede rechazar la hipótesis nula que plantea que el efecto indirecto es igual a 0 , es decir, que no existe asociación entre las variables implicadas (Hayes, 2013). En el análisis mediacional realizado se incluyeron como covariables el sexo, la edad, el autoposicionamiento político y el contacto directo con inmigrantes marroquíes para incrementar la potencia estadística de los análisis. Por tanto, con dicho análisis se responde al interrogante sobre en qué medida la identificación con el protagonista de la narración ejerce un rol mediador en la relación entre similitud y actitudes hacia la inmigración e intención de conducta (e independientemente del efecto de las variables control consideradas). 


\section{DISERTACIONES}

ESTUDIOS

Estudios de audiencias y recepción: audiencias minoritarias y nuevas mediaciones

ISSN: 1856-9536

Doi: http://dx.doi.org/10.12804/revistas.urosario.edu.co/disertaciones/v11i1

Volumen 11, Número 1 / Enero-junio 2018

Versión PDF para imprimir desde

http://revistas.urosario.edu.co/index.php/disertaciones

Tabla 2. Análisis mediacional (PROCESS, modelo 4). Efecto indirecto de la similitud en las actitudes (H3a) e intención de conducta (H3b) a través de la identificación

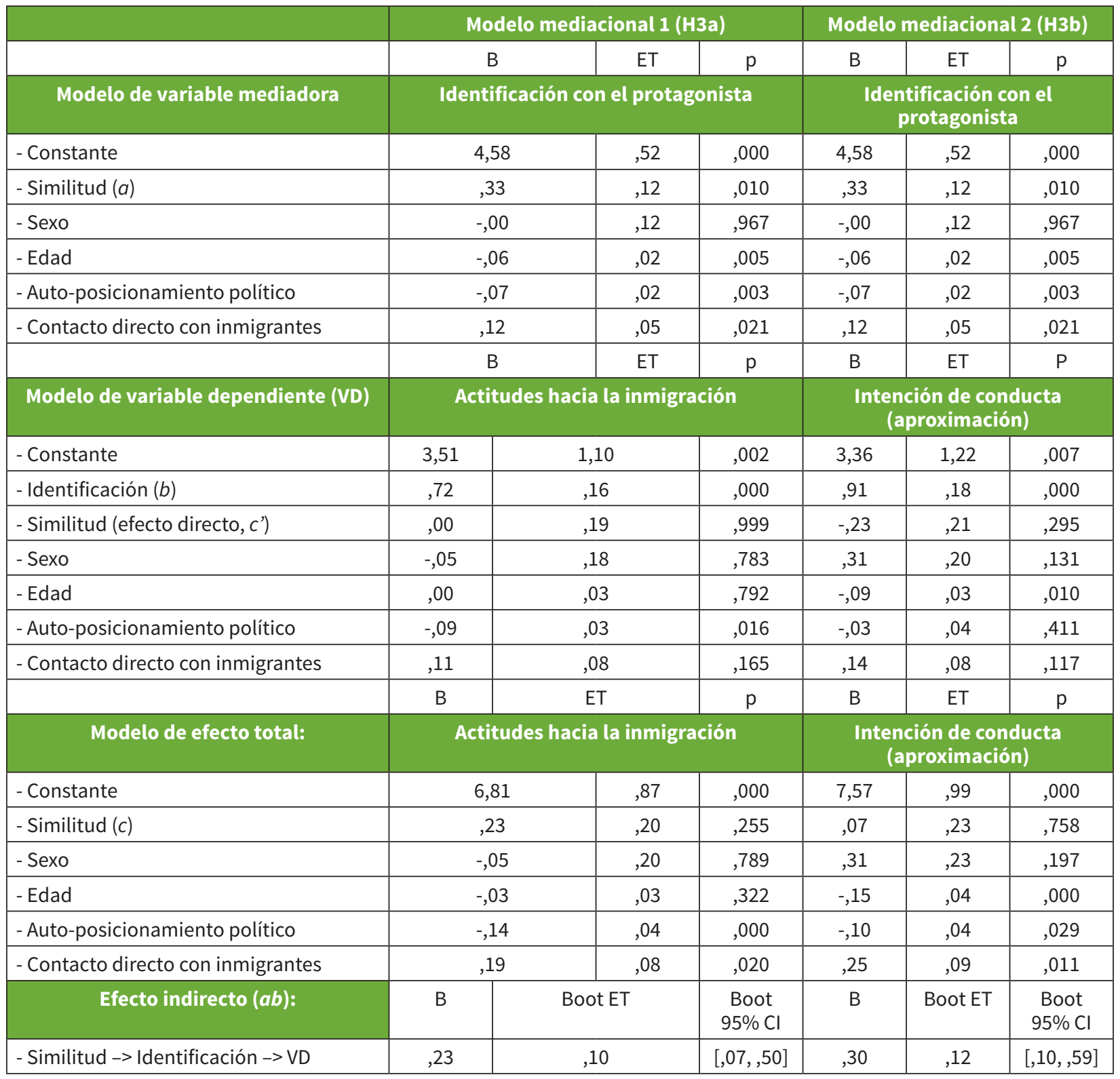

Nota: La variable independiente "similitud" fue codificada con los valores $0=$ baja similitud y $1=$ alta similitud. En la tabla se muestran los coeficientes de regresión no estandarizados (B).

Se ejecutaron dos análisis mediacionales, uno por cada variable dependiente considerada. Los resultados de los análisis (tabla 2) permitieron concluir que existía un efecto indirecto positivo y estadísticamente significativo 


\section{DISERTACIONES}

ESTUDIOS

Estudios de audiencias y recepción: audiencias minoritarias y nuevas mediaciones

ISSN: 1856-9536

Doi: http://dx.doi.org/10.12804/revistas.urosario.edu.co/disertaciones/v11i1

Volumen 11, Número 1 / Enero-junio 2018

Versión PDF para imprimir desde

http://revistas.urosario.edu.co/index.php/disertaciones

de la similitud sobre las actitudes favorables hacia la inmigración ( $B=0,23, E T=0,10,95 \% / C[0,07,0,50])$ y también sobre la intención de conducta $(B=0,30, E T=0,12,95 \% I C[0,10,0,59])$, a través de la identificación con el protagonista de la narración. Por tanto, el efecto de la similitud sobre las actitudes la intención de conducta se explica por la identificación: las personas que leyeron una narración protagonizada por un inmigrante marroquí que presentaba alta similitud en términos de identidad social con la audiencia del mensaje experimentaron una mayor identificación con el protagonista $(B=0,33, E T=0,12, p<0,010)$ lo que, a su vez, se asociaba a una actitud más favorable hacia la inmigración $(B=0,72, E T=0,16, p<0,001)$ y a una mayor intención de conducta de aproximación ( $B$ $=0,91, E T=0,18, p<0,001)$. Por tanto, los resultados del análisis mediacional suponen un apoyo empírico a la H3.

\section{Conclusiones y discusión}

Los resultados de la investigación arrojan luz sobre los procesos que explican el impacto de las narraciones protagonizadas por personajes pertenecientes a grupos estigmatizados. El trabajo se asienta teóricamente en la investigación sobre persuasión narrativa, tradición que se dedica al análisis de los procesos o mecanismos que explican el impacto de las narraciones en las personas. En este caso, se analizó el papel de la identificación con los personajes y, además se analizó el impacto de la similitud en términos de identidad social como un factor que podría incrementar la identificación con el protagonista de la narración e, indirectamente, facilitar el impacto en las medidas actitudinales.

El presente trabajo supone una novedad e innovación con respecto a trabajos previos por dos razones. En primer lugar, porque las investigaciones previas sobre persuasión narrativa han manipulado la similitud en términos demográficos (Chen et al., 2016; de Graaf, 2014; Hoeken et al., 2016; Tal-Or \& Tsfati, 2016). En cambio, en el presente trabajo se ha manipulado la similitud en términos de identidad social, es decir, en la narración protagonizada por un inmigrante se hacía explícito que dicha persona compartía rasgos relacionados con el sentimiento nacional o la apreciación de la cultura del país de acogida para incrementar la similitud con la audiencia del mensaje. En segundo lugar, los estudios previos han utilizado narraciones en las que los protagonistas de las mismas no pertenecían a grupos socialmente estigmatizados, con lo cual no enfrentaron el reto que supone tratar de estimular la identificación con un personaje que, de entrada, podría ser valorado negativamente por una parte de la audiencia. En este sentido, la investigación previa ha documentado que disponer de información positiva sobre un personaje antes de la exposición a la narración incrementa la identificación (Tal-Or \& Cohen, 2010). Igualmente, se ha observado que los personajes estigmatizados provocan menor identificación (Chung \& Slater, 2013; Igartua \& Frutos, 2017). Se plantearon tres hipótesis, dos de las cuales recibieron soporte empírico.

La primera hipótesis planteaba que la similitud entre el protagonista de la narración y la audiencia autóctona que leyó dicho mensaje influiría positivamente en la identificación con dicho personaje. Los resultados obtenidos muestran que los participantes que leyeron la narración en la que el protagonista presentaba una mayor similitud con la audiencia del menaje (con la idea de generar la sensación de compartir una identidad social común), se identificaban más con dicho personaje que los participantes que leyeron la narración en la que el protagonista no era similar. Estos resultados coinciden con estudios previos (de Graaf, 2014; Hoeken et al., 2016), aunque en dichos trabajos los protagonistas de las narraciones no pertenecieran a grupos estigmatizados como sucedió en el presente estudio, lo que resulta de gran interés de cara al diseño de campañas antiracismo teniendo en cuenta la 


\section{DISERTACIONES}

ESTUDIOS

Estudios de audiencias y recepción: audiencias minoritarias y nuevas mediaciones

ISSN: 1856-9536

Doi: http://dx.doi.org/10.12804/revistas.urosario.edu.co/disertaciones/v11i1

Volumen 11, Número 1 / Enero-junio 2018

Versión PDF para imprimir desde

http://revistas.urosario.edu.co/index.php/disertaciones

evidencia empírica sobre la dificultad de provocar identificación con personajes estigmatizados (Chung \& Slater, 2013; Igartua \& Frutos, 2017; Igartua \& Marcos, 2015).

La hipótesis 2 buscaba comprobar si la similitud del protagonista con la audiencia influía en las actitudes favorables hacia la inmigración y en la intención de conducta a interactuar con un inmigrante marroquí en el futuro. Los resultados no confirmaron dicha hipótesis, ya que los participantes que leyeron la narración en la que el protagonista presentaba una alta similitud no mostraron actitudes más positivas hacia la inmigración ni una mayor intención de conducta que los participantes que leyeron la narración en la que el protagonista no era similar a ellos. Ahora bien, el hecho de que la similitud con el protagonista no fuera un factor que tuviera un efecto total significativo en las actitudes hacia la inmigración e intención de conducta, no significa que no pudiera aumentar el impacto narrativo de manera indirecta, a través de la identificación con los personajes, tal como se había hipotetizado (H3). Como ya se mencionó en el apartado de resultados, el efecto indirecto (que representa el efecto que una variable independiente ejerce en una variable dependiente a través de una variable mediadora) puede ser estadísticamente significativo a pesar de que no se haya obtenido un efecto total significativo (Hayes, 2013).

La hipótesis 3 pretendía analizar el papel de la identificación como variable mediadora del efecto de la similitud sobre las actitudes hacia la inmigración y la intención de conducta. Los resultados de los análisis mediacionales demostraron que, efectivamente, existía un efecto indirecto estadísticamente significativo de la similitud tanto en las actitudes hacia la inmigración como en la intención de conducta y que, por tanto, la identificación con el personaje protagonista de la narración constituía una variable mediadora relevante, lo que supone un apoyo para la hipótesis 3. Dicho de otro modo, la similitud no solo incrementó la identificación, sino que además provocó una mejora en las actitudes hacia la inmigración, así como una mayor intención de conducta.

El presente estudio experimental también presenta limitaciones que deberían tenerse en cuenta de cara a futuras investigaciones. En primer lugar, se ha presentado un único estudio experimental realizado con una sola narración centrada en un solo grupo estigmatizado. Es necesario que se realicen estudios similares centrados en otros colectivos estigmatizados (por ejemplo, personas con viH, personas con obesidad, etc.), y que dichas investigaciones sean desarrolladas también en otros contextos culturales, con lo cual se ganará en validez externa. Recientemente se ha planteado como una limitación importante de la investigación en psicología de medios (media psychology) la realización de investigaciones experimentales con un solo estímulo, a pesar de que el 65\% de los estudios de este campo utilizan un solo ejemplar para representar el conjunto de la población de mensajes mediáticos (Reeves, Yeykelis, \& Cummings, 2016).

Una segunda limitación está relacionada con la muestra de participantes: estudiantes universitarios. Conviene contrastar en el futuro el papel de la similitud y la identificación con personajes estigmatizados con otro tipo de poblaciones, donde la presencia de actitudes de rechazo hacia grupos estigmatizados será mayor dado que la educación se convierte en un factor protector contra la intolerancia y el prejuicio hacia inmigrantes (Cea D’Ancona \& Valles Martínez, 2014).

En tercer lugar, el presente proyecto no contempló el papel de variables moderadoras. En este sentido, la tendencia actual en psicología de medios consiste en incluir en un mismo estudio variables mediadoras -en el presente estudio, la identificación-y moderadoras relacionadas con las diferencias individuales - por ejemplo, el nivel de racismo moderno de los participantes - (Valkenburg, Peter, \& Walther, 2016). Desde esta perspectiva se asume que los efectos mediáticos deben analizarse como procesos condicionales, que los procesos de recepción 


\section{DISERTACIONES}

ESTUDIOS

deben considerarse como variables mediadoras y que existen variables disposicionales, evolutivas y socioculturales que condicionan el efecto de la exposición a los mensajes mediáticos en las variables de resultado (Valkenburg \& Peter, 2013).

Finalmente, la investigación futura en este campo debería considerar otras dimensiones que pudieran influir en la construcción de personajes, y también otras variables textuales (como la voz narrativa) o audiovisuales (como la escala de planos o la profundidad de campo), que pudieran tener una incidencia positiva en la identificación. En este sentido, la investigación realizada abre una línea de estudio sobre los factores vinculados con la construcción de los personajes y mensajes narrativos, y su incidencia en procesos como la identificación, el transporte o enganche narrativo, e indirectamente, en variables actitudinales vinculadas con el cambio social y la promoción de la tolerancia.

\section{Referencias}

1. Braddock, K., \& Dillard, J. P. (2016). Meta-analytic evidence for the persuasive effect of narratives on beliefs, attitudes, intentions, and behaviors. Communication Monographs, 83(4), 446-467.

2. Cea D’Ancona, M. Á., \& Valles Martínez, M. (2014). Evolución del racismo, la xenofobia y otras formas conexas de intolerancia en España [Informe-Encuesta 2014]. Madrid: Oberaxe, Ministerio de Empleo y Seguridad Social.

3. Chen, M., Bell, R. A., \& Taylor, L. (2016). Narrator point of view and persuasion in health narratives. The role of protagonist-reader similarity, identification, and self-referencing. Journal of Health Communication, 21(8), 908-918.

4. Cho, H., Shen, L., \& Wilson, K. (2014). Perceived realism: Dimensions and roles in narrative persuasion. Communication Research, 41(6), 828-851.

5. Chung, A. H., \& Slater, M. D. (2013). Reducing stigma and out-group distinctions through perspective-taking in narratives. Journal of Communication, 35(3), 442-463.

6. CIS. (2014). Actitudes hacia la inmigración (Estudio no 3019). Recuperado de http://www.cis.es/cis/opencm/ ES/1_encuestas/estudios/ver.jsp?estudio=14056

7. Cohen, J. (2001). Defining identification: a theoretical look at the identification of audiences with media characters. Mass Communication \& Society, 4(3), 245-264.

8. Cohen, J., Tal-Or, N., \& Mazor-Tregerman, M. (2015). The tempering effect of transportation: Exploring the effects of transportation and identification during exposure to controversial two-sided narratives. Journal of Communication, 65(2), 237-258.

9. Crisp, R. J., \& Husnu, S. (2011). Attributional processes underlying imagined contact effects. Group Processes \& Intergroup Relations, 14(2), 275-287.

10. Frank, L. B., Murphy, S. T., Chatterjee, J. S., Moran, M. B., \& Baezconde-Garbanati, L. (2015). Telling stories, saving lives: creating narrative health messages. Health Communication, 30(2), 154-63.

11. de Graaf, A. (2014). The effectiveness of adaptation of the protagonist in narrative impact: similarity influences health beliefs through self-referencing. Human Communication Research, 40(1), 73-90. 


\section{DISERTACIONES}

ESTUDIOS

12. de Graaf, A., Hoeken, H., Sanders, J., \& Beentjes, J. W. J. (2012). Identification as a mechanism of narrative persuasion. Communication Research, 39(6), 802-823.

13. de Graaf, A., Sanders, J., \& Hoeken, H. (2016). Characteristics of narrative interventions and health effects: a review of the content, form, and context of narratives in health-related narrative persuasion research. Review of Communication Research, 4, 88-131.

14. Green, M. C., \& Brock, T. C. (2000). The role of transportation in the persuasiveness of public narratives. Journal of Personality and Social Psychology, 79(5), 701-721.

15. Hayes, A. F. (2013). Introduction to mediation, moderation, and conditional process analysis. A regression-based approach. Nueva York: Guilford Press.

16. Hoeken, H., \& Fikkers, K. M. (2014). Issue-relevant thinking and identification as mechanisms of narrative persuasion. Poetics, 44, 84-99.

17. Hoeken, H., Kolthoff, M., \& Sanders, J. (2016). Story perspective and character similarity as drivers of identification and narrative persuasion. Human Communication Research, 42(2), 292-311.

18. Igartua, J. J. (2010). Identification with characters and narrative persuasion through fictional feature films. Communications. The European Journal of Communication Research, 35(4), 347-373.

19. Igartua, J. J., \& Barrios, I. (2012). Changing real-world beliefs with controversial movies: processes and mechanisms of narrative persuasion. Journal of Communication, 62(3), 514-531.

20. Igartua, J. J., \& Frutos, F. J. (2017). Enhancing attitudes toward stigmatized groups with movies: Mediating and moderating processes of narrative persuasion. International Journal of Communication, 11, 158-177.

21. Igartua, J. J., \& Marcos, M. (2015). Influence of character type and narrative setting on character design for fictional television series. Communication \& Society, 28(1), 63-77.

22. Igartua, J. J., \& Vega, J. (2016). Identification with characters, elaboration, and counterarguing in entertainment-education interventions through audiovisual fiction. Journal of Health Communication, 21(3), 293-300.

23. Kaufman, G. F., \& Libby, L. K. (2012). Changing beliefs and behavior through experience-taking. Journal of Personality and Social Psychology, 103(1), 1-19.

24. Moyer-Gusé, E. (2008). Toward a theory of entertainment persuasion: explaining the persuasive effects of entertainment-education eessages. Communication Theory, 18(3), 407-425.

25. Müller, F. (2009). Entertaining anti-racism. Multicultural television drama, identification and perceptions of ethnic threat. Communications, 34(3), 239-256.

26. Murphy, S. T., Frank, L. B., Chatterjee, J. S., \& Baezconde-Garbanati, L. (2013). Narrative versus nonnarrative: the role of identification, transportation, and emotion in reducing health disparities. Journal of Communication, 63(1), 116-137.

27. Ortiz, M., \& Harwood, J. (2007). A social cognitive theory approach to the effects of mediated intergroup contact on intergroup attitudes. Journal of Broadcasting \& Electronic Media, 51(4), 615-632.

28. Park, S.-Y. (2012). Mediated intergroup contact: concept explication, synthesis, and application. Mass Communication and Society, 15(1), 136-159.

29. Pettigrew, T. F., \& Tropp, L. R. (2006). A meta-analytic test of intergroup contact theory. Journal of Personality and Social Psychology, 90(5), 751-783. 
30. Reeves, B., Yeykelis, L., \& Cummings, J. J. (2016). The use of media in media psychology. Media Psychology, 19(1), 49-71.

31. Schiappa, E., Gregg, P. B., \& Hewes, D. E. (2005). The parasocial contact hypothesis. Communication Monographs, 72(1), 92-115.

32. Slater, M. D., \& Rouner, D. (2002). Entertainment-education and elaboration likelihood: understanding the processing of narrative persuasion. Communication Theory, 12(2), 173-191.

33. Tal-Or, N., \& Cohen, J. (2010). Understanding audience involvement: conceptualizing and manipulating identification and transportation. Poetics, 38(4), 402-418.

34. Tal-Or, N., \& Tsfati, Y. (2016). When Arabs and Jews watch TV together: the joint effect of the content and context of communication on reducing prejudice. Journal of Communication, 66(4), 646-668.

35. Valkenburg, P. M., \& Peter, J. (2013). The differential susceptibility to media effects model. Journal of Communication, 63(2), 221-243.

36. Valkenburg, P. M., Peter, J., \& Walther, J. B. (2016). Media effects: theory and research. Annual Review of Psychology, 67, 315-338.

37. Wojcieszak, M., \& Kim, N. (2016). How to improve attitudes toward disliked groups: the effects of narrative versus numerical evidence on political persuasion. Communication Research, 43(6), 785-809. 